\title{
Técnicas de aprendizaje virtual para estudiantes con dificultades atencionales
}

\section{Virtual learning techniques for students with attention difficulties}

1 Yamilec Alexandra López Bermello

(iD https://orcid.org/0000-0002-5813-8315

Universidad Técnica de Ambato, Facultad de Ciencias Humanas y de la Educación,

Carrera de Psicopedagogía, Ambato, Ecuador, Ecuador.

ylopez5558@uta.edu.ec.

2 Estefanía Brigitte Gamboa Cuatimpaz (iD) https://orcid.org/0000-0002-4161-0783

Universidad Técnica de Ambato, Facultad de Ciencias Humanas y de la Educación,

Carrera de Psicopedagogía, Ambato, Ecuador, Ecuador.

egamboa5782@uta.edu.ec.

3 Joel Alejandro Cuesta Estupiñán (iD) https://orcid.org/0000-0003-2885-5556

Universidad Técnica de Ambato, Facultad de Ciencias Humanas y de la Educación,

Carrera de Psicopedagogía, Ambato, Ecuador, Ecuador.

jestupinan0403@uta.edu.ec.

4 María Fernanda Morales Gómez de la Torre (iD) https://orcid.org/0000-0002-4780-0596

Uniandes, Facultad de Ciencias de la salud, Carrera de Enfermería, Ambato, Ecuador.

ua.mariamorales@uniandes.edu.ec

\section{Artículo de Investigación Científica y Tecnológica}

Enviado: 24/12/2021

Revisado: 29/12/2021

Aceptado: $12 / 01 / 2022$

Publicado:08/03/2023

DOI: https://doi.org/10.33262/concienciadigital.v6i1.4.2006

Cítese: López Bermello, Y. A., Gamboa Cuatimpaz, E. B., Cuesta Estupiñán, J. A., \& Morales Gómez de la Torre, M. F. (2023). Técnicas de aprendizaje virtual para estudiantes con dificultades atencionales. ConcienciaDigital, 6(1.4), 417-436. https://doi.org/10.33262/concienciadigital.v6i1.4.2006

CONCIENCIA DIGITAL, es una Revista Multidisciplinar, Trimestral, que se publicará en soporte electrónico tiene como misión contribuir a la formación de profesionales competentes con visión humanística y crítica que sean capaces de exponer sus resultados investigativos y científicos en la misma medida que se promueva mediante su intervención cambios positivos en la sociedad. https://concienciadigital.org .

La revista es editada por la Editorial Ciencia Digital (Editorial de prestigio registrada en la Cámara Ecuatoriana de Libro con No de Afiliación 663) www.celibro.org.ec 


\section{Palabras}

claves:

educación, aprendizaje virtual, dificultades atencionales, estudiantes.

\section{Keywords:}

education, virtual learning, attention difficulties, students.

\section{Resumen}

Introducción. En la educación siempre han existido condiciones que interrumpen el aprendizaje de los estudiantes uno de ellos es la dificultad atencional, la cual radica en el déficit para prestar atención durante un periodo largo de tiempo en acciones determinadas como al realizar tareas o atender a clases, esto se debe que la atención en un proceso compuesto por varios componentes que interactúan entre sí. Por ende, es necesaria la aplicación de varias técnicas de aprendizaje virtual para que los alumnos puedan llegar a consolidar la información y de esta manera obtengan un adecuado rendimiento académico. Objetivo: Analizar la influencia de las técnicas de aprendizaje virtual para estudiantes con dificultades atencionales durante el proceso académico en línea. Metodología. Se aplica un enfoque mixto ya que se busca analizar, describir la relación de las técnicas de aprendizaje virtual con los estudiantes con dificultades atencionales, un diseño descriptivo, una modalidad de campo y bibliográfica ya que la información fue recolectada de fuentes primarias. Resultados. Entre los resultados más relevantes se tiene que los estudiantes presentan dificultades atencionales esto debido a varios factores como la educación en línea y los diferentes distractores que se encuentran en sus hogares. Conclusión. La implementación y aplicación de métodos adecuados en las clases virtuales son necesarios para un correcto desempeño en los estudiantes, así como para focalizar la atención de estos y de esa manera obtener resultados eficaces en el aprendizaje en línea.

\section{Abstract}

Introduction. In education there have always been conditions that interrupt the learning of students, one of them is the attentional difficulty, which lies in the deficit to pay attention for a long period of time in certain actions such as performing tasks or attending classes, this It is due to the attention in a process composed of several components that interact with each other. Therefore, it is necessary to apply various virtual learning techniques so that students can consolidate the information and thus obtain adequate academic performance. Objective: To analyze the influence of virtual learning techniques for students with attention difficulties during the online academic process. Methodology. A mixed approach is applied since it seeks to analyze, describe the 
relationship of virtual learning techniques with students with attentional difficulties, a descriptive design, a field and bibliographic modality since the information was collected from primary sources. Results. Among the most relevant results, students have attentional difficulties due to several factors such as online education and the different distractions found in their homes.

Conclusion. The implementation and application of adequate methods in virtual classes are necessary for a correct performance in students, as well as to focus their attention and thus obtain effective results in online learning.

\section{Introducción}

Se ha logrado constatar que a causa de la pandemia se ha visto afectado el proceso de aprendizaje de los alumnos y sobre todo en aquellos quienes presentan cierto tipo de dificultad para atender a clases o a su vez no prestan toda su atención a lo que dice el profesor o de igual manera cuando no se concentra al realizar sus actividades académicas. Por tanto, resulta fundamental que el docente emplee herramientas tecnológicas que ayuden a la consolidación del aprendizaje de los estudiantes, siendo así que en la presente investigación se da a conocer sobre actividades imprescindibles que deben ser aplicadas en clases con la finalidad de que permitan a los estudiantes participar plenamente en los procesos pedagógicos, alcanzar los conocimientos y temáticas propuestas y así lograr en ellos un aprendizaje significativo.

\section{Aprendizaje Virtual}

El aprendizaje virtual supone un cambio en la consolidación del conocimiento y la información a través de sistemas inteligentes, los cuales permiten describir el contenido de los materiales educativos utilizados, brindando una interacción en línea entre docentes y estudiantes a través de plataformas digitales. En otras palabras, el sujeto educativo pasa de ser consumidor a productor de información; el rol explicativo del docente ahora es comprendido como guía ya que brinda la enseñanza pertinente por medio de estrategias y técnicas para favorecer el aprendizaje tecnológicamente mediado (Aguilar, 2020).

Con el pasar del tiempo todo va avanzando y por ello cada vez más son los beneficios que facilitan los procesos de aprendizaje virtual, por ende, el acceso inmediato a las fuentes de información a internet, la oportunidad de repetir las clases grabadas en zoom o teams, el ahorro de dinero en transportes y costos que implica el asistir a las instituciones educativas y la seguridad de estudiar desde casa implica que se evidencien aspectos positivos en dicha modalidad (Navarrete et al., 2019). 
Por otro lado, el hecho de recibir las clases por medio de dispositivos electrónicos ha dado paso a grandes dificultades en los estudiantes como son los problemas de conectividad, la falta de equipos informáticos, limitaciones en relaciones de aprendizaje compartido de manera presencial, aumento de la distraibilidad lo que trae consigo la perdida de atención en los alumnos lo que ha dado paso a varias afectaciones a nivel psicológico, social y educativo lo que ha perjudicado en el desarrollo integral de los mismos (Jaime Torres, 2019).

\section{Retos del Aprendizaje Virtual}

Dadas las circunstancias que se viven hoy en día, se han tenido que cerrar varias instalaciones poniendo como una forma de solución los medios digitales para lograr comunicarnos con los demás, sin embargo, esto ha traído consigo varias repercusiones como facilidad de acceso a dispositivos electrónicos, brechas digitales e incluso con afectaciones psicológicas como ansiedad, depresión y demás por el hecho de poder pasar solo en casa, todo lo anterior conduce a enfatizar la realidad que sin duda ha sido y es el hecho de adaptarse y entender las necesidades de cada uno con la finalidad de llevar procesos adecuados que no perjudiquen al desenvolvimiento cotidiano de las personas (Valero Cedeño, 2020).

Cabe considerar por otra parte que, de gran manera los más susceptibles a estos cambios son aquellos que presentan alguna discapacidad debido a que ellos requieren de mayor atención y apoyo, por tanto se suman las barreras educativas que limitan el acceso a programas tecnológicos, igualdad de oportunidades y brechas digitales, de manera que esos aspectos no generan muchos avances en dichos grupos, sin embargo, se puede mencionar que con el labor arduo y constante de profesores y familiares se logra que el estudiante con alguna NEE logre de a poco incluirse en los espacios virtuales de aprendizaje (Peña Estrada, 2020).

\section{Entornos virtuales en el aprendizaje}

Los entornos virtuales de aprendizaje dan paso a que el acceso a contenidos educativos sea más flexible y que a su vez se combinen varios recursos, lo que significa un reto tanto para docentes pues deben brindar un ambiente adecuado de aprendizaje por medio de espacios dinámicos y participativos para unas clases enriquecedoras y que asimismo sean motivantes para los estudiantes de manera que lleguen a integrar todos los conocimientos, habilidades y valores que les permitan resolver tareas con mayor productividad (Cortes y Sánchez, 2021).

En la medida que el tiempo avanza todo ha ido cambiado, por ende los alumnos se han ido adaptando al estilo de vida tecnológica siendo así que los docentes por medio de una constante preparación y formación se vieron en la necesidad de acoplar la enseñanza 
virtual según las características, ritmo y estilo de aprendizaje de los alumnos por medio de diversidad herramientas de las TIC como: correo electrónico, foros, videoconferencias, plataformas telemáticas, entre otras; las cuales son importantes para el cumplimiento de tareas y sobre todo para facilitar la construcción de saberes y generar procesos de formación eficaces que permitan el desenvolvimiento pleno de los alumnos (Espinoza Freire, 2018).

\section{Técnicas de Aprendizaje Virtual en estudiantes}

Según Delgado (2021) La integración de las herramientas tecnológicas ha facilitado de manera significativa los progresos de la educación a distancia, haciendo especial énfasis en los recursos virtuales como la utilización de Zoom o Teams que son herramientas importantes para que los docentes impartan sus clases y además de ello buscan potenciar el aprendizaje por medio de la aplicación de varias técnicas como: glosarios colaborativos, los cuales permiten el trabajo en equipo y desarrollan el pensamiento crítico; subgrupos de discusión, para debatir sobre algún tema en específico; también está la exposición didáctica, que consiste en la presentación de un tema por parte del docente o alumnos apoyándose en elementos gráficos y visuales; lluvias de ideas, en donde el docente da un tema y los alumnos van aportando con ideas, así mismo la tutoría publica, aquí el profesor brinda a los alumnos un espacios de conferencia electrónica con el fin de aclarar dudas y mencionar otros asuntos varios y también se tiene al portafolio que es aquella plataforma online primordial para que el estudiante suba sus tareas, participe en foros y realice actividades en beneficio a su formación académica.

En concordancia con los aspectos mencionados anteriormente es preciso mencionar de igual forma a las pausas activas que el docente debe manejar con los estudiantes por medio de breves descansos durante las clases para mejorar la participación y desempeño de los mismos, así como también las cámaras encendidas, también el estar en continua conversación con cada estudiante y sobre brindar información de introducción a cada inicio de modulo para herramientas básicas como la técnica pomodoro que permite administrar el tiempo de trabajo, planificar y ejecutar tareas para que los estudiantes mejoren su productividad durante 25 minutos de tal forma que se concentren y logren culminar una tarea en particular en ese tiempo (Restrepo Echavarría, 2018).

No cabe duda de que todas las técnicas mencionadas con anterioridad resultan importantes para promover la motivación en los alumnos y que de esa manera sus conocimientos sean consolidados. Además, existen otros apoyos que se deben tomar en cuenta y son: rueda de ideas, apuntes de grupo, controversia estructurada, presentación de videos, etc. Siendo así que dichas técnicas conllevan a un adecuado desarrollo del currículo propuesto a nivel educativo proporcionando así grandes ventajas al proceso enseñanza y aprendizaje mediado por tecnologías. 


\section{Importancia de los recursos tecnológicos para el aprendizaje virtual}

La influencia de la tecnología en el ámbito educativo en estos tiempos cumple un rol fundamental en todos los niveles y modalidades de los sistemas pedagógicos puesto a que ha proporcionado la facilidad de utilizar varios recursos disponibles para el desarrollo y mejora en alumnos con dificultades atencionales, pues por medio de la utilización de juegos virtuales como herramientas digitales optimizan el nivel de concentración y atención mejorando su desenvolvimiento escolar brindando así mismo el aspecto motivacional en la persona para que de esa manera pueda continuar con sus estudios. En última instancia este tipo de medios digitales benefician a los estudiantes con dificultades atencionales ya que, al ser accesibles y flexibles, promueven a que los profesores puedan adaptar la práctica educativa de acuerdo con contenido y objetivos teniendo en cuenta las necesidades del alumnado que padece esta dificultad (Guerrero y González, 2021).

Cabe destacar que, el docente se ve en la necesidad de implementar una fuente de estrategias encaminadas a la motivación del alumno para captar su atención en clases, por eso se debe promover la participación activa, así como el uso de contenidos multimedia en las presentaciones explicativas de contenidos específicos y asimismo el hecho de enfatizar las interacciones por medio de diálogos o espacios grupales de aprendizaje, ya que con todo ello se busca lograr un ambiente propicio para la formación académica (Gavilanes et al., 2019).

\section{Dificultades atencionales Atención}

La atención es la capacidad de seleccionar y concentrarse en los estímulos, Esto quiere decir que la atención es el proceso cognitivo que nos permite orientar los estímulos, lograr procesarlos para responder en acciones de la vida diaria, Cabe destacar que la atención no es un proceso unitario, sino que existen distintos tipos de atención. En resumen, se puede decir que la atención es una capacidad que nos sirve para crear, guiar y mantener nuestro cerebro activo de manera que podamos procesar correctamente la información (Martínez y Yévenes, 2020).

\section{Atención y aprendizaje}

La atención es fundamental en la educación ya que intervienen en la cognición y en las acciones humanas, ya que responsabiliza en la activación de los procesos de estímulos específicos necesarios para llevar a cabo cualquier tarea, por ello, en un contexto educativo, los adolescentes reciben estímulos de los cuales pueden extraer información de forma clara y concisa. Por otra parte, una de las principales preocupaciones por parte de los docentes, en la presencia de problemas relacionados con la atención, al considerarse la base para el desarrollo de otros procesos cognitivos y la influencia en el proceso de enseñanza-aprendizaje es innegable la presencia de atención (Mattiooli, 2019). 


\section{Dificultades atencionales}

En nuestro entorno siempre existirán un tipo de dificultades especialmente en la atención, este problema se puede apreciar en situaciones cotidianas como, por ejemplo: La explicación de un tema en clases, mientras se mantienen una conversación con otros o en relacionada a tareas simultaneas, así pues, el termino dificultad hace referencia a la limitación, discapacidad o deficiencia que presenta una persona al realizar una acción o grupo de acciones que podrían provocar su mal cumplimiento (Ramírez et al., 2020).

Las Dificultades atencionales serán unos de los problemas más comunes que se pueden encontrar en la educación, la DA se caracteriza en que los estudiantes presentan distraibilidad, ensimismamiento, dificultad para identificar y prestar atención. Estas dificultades en los adolescentes suelen ser muy significativas debido a la relación que se presenta en su rendimiento. El DA es fácilmente detectable si los alumnos presentan incapacidad para controlar sus respuestas en una acción determinada, presentan dificultades para inhibir las respuestas espontaneas, falta de atención y concentración, tienen dificultades tanto para controlar su conducta como para controlar sus emociones y para controlar sus pensamientos (Liesa et al., 2017).

\section{Tipos de Dificultades atencionales}

Según el DSM-V, las dificultades atencionales no afectan a toda la población por igual. Así, se pueden diferenciar dos grandes grupos de acuerdo con dos variables dentro de los tipos se encuentran Dificultad atencional en la atención sostenida (AS) y dificultad en la calidad atencional (CA).

\section{Tabla 1}

\section{Dificultad atencional}

\begin{tabular}{ll}
\hline Dificultad atencional en la atención sostenida (as) & Dificultad en la calidad atencional (ca) \\
\hline Estos presentan un déficit en la atención & Se caracterizan por presentar distracción, \\
sostenida, pueden ser reconocidos por atender a & ensimismamiento y una clara dificultad para \\
un estímulo durante un periodo corto de tiempo, & identificar el estímulo que es relevante \\
mostrando cambios de atención. &
\end{tabular}

Fuente: Asociación Americana de Psiquiatría (2013).

Factores de riesgo

Los factores de riesgo son condiciones, conductas o situaciones de la vida que exponen a tener un mayor riesgo de presentar cierta enfermedad, es por esto por lo que es más posible padecer este trastorno conociendo los diferentes factores presentes, Entre mayor sean los riesgos que están presentando mayores serán las probabilidades de que el niño o 
adolescente (Martinhago et al., 2019).

Entre los factores de riesgo más frecuentes encontramos.

Familiares que pueden ser consanguíneos.

$>$ Exposición a tóxicos ambientales, como el plomo, dióxido de carbono, estos tóxicos se pueden encontrar en pinturas, tuberías, humo de carros.

$>$ Consumo de drogas, por ejemplo, alcohol, tabaco o sustancias psicotrópicas.

$>$ Nacimiento prenatal, accidentes en el embarazo, estrés o consumo de pastillas o medicamentos inadecuados en el desarrollo del embarazo (Chacón et al., 2018).

\section{Afectación Emocional}

Los estudios de las emociones en un contexto educativo tienen un gran peso actualmente, debido a las diferentes investigaciones científicas en relación con este tema, dependen total mente de la relación de las emociones y la educación forman a si una solo fuerza que impulsan y contribuyen a lo largo de sus objetivos que se proponen los estudiantes, sobre todo en aquellos que inician su etapa de escolarización, o en niños que presentan alguna dificultad académica (Valverde et al., 2020).

La importancia de los factores emocionales en el desarrollo de los alumnos con DA, es muy importante ya que pueden aportar un autocontrol, además logran dar capacidades de motivación a lo largo del tiempo, logrando conseguir objetivos propuestos, también nos permite inhibir emociones fuertes, modificarlas, distraerlos. Es por estas razones que las emociones juegan un papel importante en el desarrollo educativo de niños Con DA (González et al., 2020).

\section{Las dificultades atencionales y el aprendizaje significativo}

Según la relación entre los síntomas que presentan el DA y el aprendizaje escolar en los estudiantes determina que la atención, dentro de las tres características que puede tener una persona con dicha condición (inatención, impulsividad e hiperactividad), es la que se asocia principalmente con el rendimiento académico. A más de ello existen causas como la utilización de métodos tradicionales al impartir las clases y de igual manera al realizar las evaluaciones sobre temas específicos, por tanto, se debe tomar en cuenta la utilización de los medios que brinda la tecnología para una mayor efectividad en el aprendizaje de los estudiantes, así como para que se logre una mejor consolidación de la información de los educandos (González et al., 2020).

Todo esto hace suponer que no son las dificultades conductuales sino más bien es por la problemática atencional, la cual es un factor importante que infiere en el proceso de 
aprendizaje; ahora bien el déficit atencional suele generar problemas en el aprendizaje que en ciertos casos de personas con mayor edad, quienes han vivido con deficiencias educativas, tal vez hubieran podido mejorar su situación si en algún caso se hubieran diagnosticado a tiempo y así mismo hubieran tenido una intervención adecuada, la cual le ayude a mantener e incluso a mejorar su nivel y rendimiento académico, para que en un futuro pueda servirle todo lo que se le haya aplicado (Cedeño y Rodríguez, 2019).

\section{Estrategias Educativas para trabajar con Universitarios con Dificultades atencionales}

Dentro de cualquier institución es fundamental que el profesorado introduzca adaptaciones ambientadas y metodológicas encargadas de beneficiar a los alumnos con DA así como al resto del grupo, por ello, cabe mencionar que el profesor al ser el guía en el proceso de enseñanza debe ser quien motive a sus estudiantes promoviendo la participación activa de manera que ellos sean los protagonistas, por ende, hay una gran variedad de actividades que pueden servir a los docentes como guía de ayuda para sus estudiantes, tratándose de reducir el nivel de exigencia sino de modificar las condiciones en las que se desarrolla la clase sobre todo en la modalidad que se está manejando en estos momentos (Quila et al., 2020).

A continuación, se mencionan una serie de estrategias que serán muy útiles tanto de manera organizativa como metodológica puesto a que las mismas ayudarán a controlar la atención:

La organización de tareas: Uno de los primeros pasos para lograr que los estudiantes mejorar su atención es permitirles que organicen sus tareas antes de cada clase esto.

$>$ Proponer actividades significativas para los jóvenes, esto implica dar actividades relacionadas con sus conocimientos previos o que tengan que ver con su vida cotidiana y que le susciten interés.

$>$ Fomentar más el trabajo en equipo y el trabajo conjunto con el profesor mediante las salas de Zoom o Teams.

$>$ Proporcionar el aprendizaje dialógico: grupos interactivos, tertulias, etc.

$>$ Aplicación de clases con instrumentos audiovisuales que llamen la atención de los adolescentes.

$>$ Hacer pausas de 5 minutos para aumentar la relajación de los estudiantes (Suárez et al.,2018).

Actividades lúdicas que mejoran el aprendizaje en estudiantes con Dificultades atencionales

A lo largo de la vida académica es fundamental el dinamismo en las actividades en clase para que el estudiante se sienta motivado y pueda rendir de mejor manera en sus clases, 
también es posible la interacción con el grupo y a su vez una gran manera de captar la atención del alumno, al inicio o durante una clase. Por esta razón, la utilización de juegos ayuda a mejor el proceso pedagógico, y de esta manera resulte un aprendizaje agradable y dinámico, ya que mediante esta metodología es posible desarrollar habilidades motoras y capacidades como interactuar, socializar y fomentar la creatividad. Siendo así que su aplicación en la vida cotidiana posibilita la mejora de los procedimientos y técnicas por medio de los docentes, lo mismo que implica una correcta planificación basada en decisiones que el profesor debe tomar en cuenta para lograr las metas, objetivos, y propósitos propuestos del procedo educativo (Abril Iza, 2020).

Las actividades recreativas permiten la integración en todos los jóvenes, lo cual promueve sentir entusiasmo y energía, teniendo un gran impacto y favoreciendo en el desarrollo de los aspectos emocionales, cognitivos y sociales. Este tipo de actividades dentro de ámbito escolar son consideradas como herramientas, técnicas o hasta cierto punto estrategias importantes, obviamente siendo utilizadas de manera correcta ya que mediante estas ayudamos al proceso mental y el razonamiento lógico en una persona.

\section{Metodología}

En el presente estudio se aplica un enfoque mixto ya que buscamos analizar, describir la relación de las técnicas de aprendizaje virtual con los estudiantes con dificultades atencionales, un diseño descriptivo, una modalidad de campo y bibliográfica ya que la información fue recolectada de fuentes primarias.

Cuenta con una investigación descriptiva ya que está basada en una recopilación de información sobre las variables de estudio y por otra parte tiene un enfoque mixto puesto a que se tiene la utilización de técnicas de recolección de datos, por ende, en este estudio se integran métodos cualitativos como cuantitativos para brindar una mejor comprensión permitiendo entender la realidad del tema de una manera más integral.

Mediante la modalidad de campo se fue recolectando los datos de interés de forma directa en las aulas virtuales de Zoom y a través de la aplicación de dos encuestas, la primera para conocer si se emplean técnicas de aprendizaje virtual y la segunda para determinar si los alumnos presentan o no dificultades atencionales. Dichos cuestionarios fueron destinados a una muestra de 124 estudiantes de la Universidad Técnica de Ambato a los cuales se les aplicó la correlación de Spearman. Los resultados de estas encuestan permiten verificar si las técnicas de aprendizaje virtual influyen de manera positiva o negativa en los estudiantes con dificultades atencionales en su rendimiento académico. 


\section{Resultados}

\section{Tabla 2}

Escala de medición de las opciones de respuesta

\begin{tabular}{cccc}
\hline NADA & POCO & BASTANTE & MUCHO \\
\hline 0 & 1 & 2 & 3 \\
\hline
\end{tabular}

Fuente: Encuesta virtual a estudiantes de la Universidad Técnica de Ambato (2021)

\section{Tabla 3}

Cuestionario sobre técnicas de aprendizaje virtual

\begin{tabular}{|c|c|c|c|c|c|c|c|c|}
\hline $\begin{array}{l}\text { DESCRIPCIÓN } \\
\text { 1. ¿Consideras útil recibir }\end{array}$ & \multicolumn{2}{|c|}{$\mathrm{NADA}=0 \%$} & \multicolumn{2}{|c|}{$\mathrm{POCO}=1 \%$} & \multicolumn{2}{|c|}{ BASTANTE $=2 \%$} & \multicolumn{2}{|c|}{$\mathrm{MUCHO}=3 \%$} \\
\hline $\begin{array}{l}\text { 1. ¿Consideras útil recibir } \\
\text { clases mediante plataformas } \\
\text { digitales Zoom o Teams para } \\
\text { tu aprendizaje? }\end{array}$ & 6 & $5 \%$ & 48 & $39 \%$ & 47 & $38 \%$ & 23 & $19 \%$ \\
\hline $\begin{array}{l}\text { 2. ¿Te sientes motivado } \\
\text { cuando tus profesores utilizan } \\
\text { videos y presentaciones para } \\
\text { explicar los temas? }\end{array}$ & 0 & $0 \%$ & 36 & $29 \%$ & 54 & $44 \%$ & 34 & $27 \%$ \\
\hline $\begin{array}{l}\text { 3. Al iniciar una clase, ¿El } \\
\text { profesor emplea alguna } \\
\text { actividad dinámica de manera } \\
\text { que busque la participación } \\
\text { activa de todos? }\end{array}$ & 16 & $13 \%$ & 46 & $37 \%$ & 40 & $32 \%$ & 22 & $18 \%$ \\
\hline $\begin{array}{l}\text { 4. ¿Estás de acuerdo en que las } \\
\text { clases sean explicadas a través } \\
\text { de elementos multimedia } \\
\text { (animación, sonido, gráficos, } \\
\text { videos)? }\end{array}$ & 0 & $0 \%$ & 12 & $10 \%$ & 57 & $46 \%$ & 55 & $44 \%$ \\
\hline $\begin{array}{l}\text { 5. ¿Han resultado eficaces los } \\
\text { trabajos en grupo para } \\
\text { compartir información y } \\
\text { conocimiento con tus } \\
\text { compañeros? }\end{array}$ & 2 & $2 \%$ & 30 & $24 \%$ & 64 & $52 \%$ & 28 & $23 \%$ \\
\hline $\begin{array}{l}\text { 6. ¿Utilizas el chat, blog o } \\
\text { alguna otra herramienta digital } \\
\text { para tu participación en línea? }\end{array}$ & 3 & $2 \%$ & 38 & $31 \%$ & 65 & $52 \%$ & 18 & $15 \%$ \\
\hline
\end{tabular}


Tabla 3

Cuestionario sobre técnicas de aprendizaje virtual (continuación)

\begin{tabular}{|c|c|c|c|c|c|c|c|c|}
\hline $\begin{array}{l}\text { 7. ¿Estás de acuerdo en que se } \\
\text { evalúen los aprendizajes a } \\
\text { través de páginas web como: }\end{array}$ & \multicolumn{2}{|c|}{$\mathrm{NADA}=0 \%$} & \multicolumn{2}{|c|}{$\mathrm{POCO}=1 \%$} & \multicolumn{2}{|c|}{ BASTANTE $=2 \%$} & \multicolumn{2}{|c|}{$\mathrm{MUCHO}=3 \%$} \\
\hline $\begin{array}{l}\text { T. ¿Estas de acuerdo en que se } \\
\text { evalúen los aprendizajes a } \\
\text { través de páginas web como: } \\
\text { Educaplay, Socrative, } \\
\text { ¿Moodle o en la plataforma } \\
\text { digital que emplea tu } \\
\text { profesor? }\end{array}$ & 1 & $1 \%$ & 22 & $18 \%$ & 71 & $57 \%$ & 30 & $24 \%$ \\
\hline $\begin{array}{l}\text { 8. ¿Te resulta fácil subir todas } \\
\text { tus tareas o actividades a la } \\
\text { plataforma digital que utiliza } \\
\text { tu universidad? }\end{array}$ & 1 & $1 \%$ & 15 & $12 \%$ & 69 & $56 \%$ & 39 & $31 \%$ \\
\hline $\begin{array}{l}\text { 9. ¿El maestro dicta una clase } \\
\text { haciendo uso de material } \\
\text { didáctico como (pizarra o } \\
\text { diapositivas)? }\end{array}$ & 0 & $0 \%$ & 20 & $16 \%$ & 71 & $57 \%$ & 33 & $27 \%$ \\
\hline $\begin{array}{l}\text { 10. ¿Consideras que la } \\
\text { modalidad virtual ha facilitado } \\
\text { tu proceso de aprendizaje? }\end{array}$ & 19 & $15 \%$ & 60 & $48 \%$ & 37 & $30 \%$ & 8 & $6 \%$ \\
\hline TOTAL AL 100\% & & & & & & & & \\
\hline
\end{tabular}

Fuente: Encuesta virtual a estudiantes de la Universidad Técnica de Ambato

Los estudiantes participantes en la investigación indicaron que consideran útil recibir clases mediante plataformas digitales zoom o Teams para el aprendizaje con 5\% en nada, $39 \%$ poco, $38 \%$ bastante y $19 \%$ mucho. Los estudiantes dieron a conocer que se sienten motivados cuando los profesores utilizan vídeos y presentaciones para explicar los temas $0 \%$ en nada, $29 \%$ poco, $44 \%$ bastante y $27 \%$ mucho. Dentro de una clase el profesor al emplear alguna actividad dinámica de manera que busca la participación activa de todos, los estudiantes dieron a conocer que $13 \%$ nada, $37 \%$ poco, $32 \%$ bastante y $18 \%$ mucho. Los estudiantes dieron a saber que están de acuerdo en que las clases sean explicadas a través de elementos multimedia (animación, sonido, gráficos, vídeos) $0 \%$ nada, $10 \%$ poco, $46 \%$ bastante y $44 \%$ mucho. Los estudiantes dieron a conocer que han resultado eficaces los trabajos en grupo para compartir información y conocimiento con los compañeros $2 \%$ nada, $24 \%$ poco, $52 \%$ bastante y $23 \%$ mucho. Los estudiantes utilizan chat blogs o alguna otra herramienta digital para la participación en línea $2 \%$ nada, $31 \%$ poco $52 \%$ bastante y $15 \%$ mucho. Los estudiantes están de acuerdo en que se evalúen los aprendizajes a través de páginas web $1 \%$ nada, $18 \%$ poco, $57 \%$ bastante y $24 \%$ mucho. A los estudiantes se les resulta fácil subir todas sus tareas actividades de la plataforma digital que utiliza la universidad $1 \%$ nada, $12 \%$ poco, $56 \%$ bastante y $31 \%$ 
mucho. Los maestros dictan en una clase haciendo uso de material didáctico $0 \%$ nada, 16 $\%$ poco, $57 \%$ bastante y $27 \%$ mucho. Los estudiantes dijeron que la modalidad Virtual ha facilitado el proceso de aprendizaje $15 \%$ nada, $48 \%$ poco, $30 \%$ bastante y $6 \%$ mucho.

\section{Figura 1}

\section{Empleo de actividad dinámica en clase por parte del profesor}

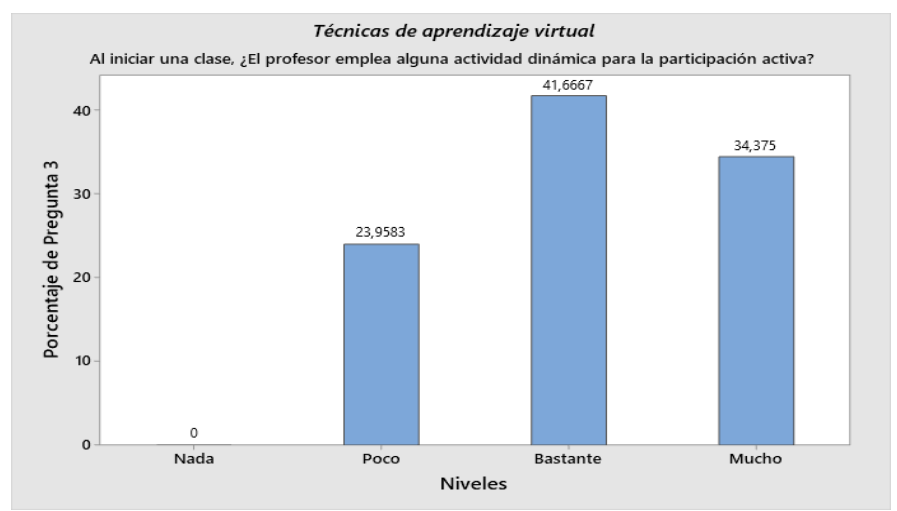

Fuente: Estudio de contexto

Luego de la aplicación del instrumento en la pregunta 3: Al iniciar la clase. ¿El profesor emplea alguna actividad dinámica para la participación activa?, las personas respondieron que el $0 \%$ de los estudiantes están en nunca, de ahí el $23 \%$ de los estudiantes que corresponden a 46 personas manifiestan que es poco lo que profesor emplea alguna actividad dinámica para la participación activa de todos, el $41 \%$ de los estudiantes que corresponde a 80 personas optaron la opción de bastante, el 34\% de los estudiantes que corresponde a 66 personas escogieron la alternativa de mucho. Por lo que se puede inferir que casi el $50 \%$ de los estudiantes están de acuerdo en que el profesor emplea alguna actividad dinámica para la participación actividad de todos en clases.

\section{Figura 2}

\section{Evaluación de aprendizaje a través de páginas web}

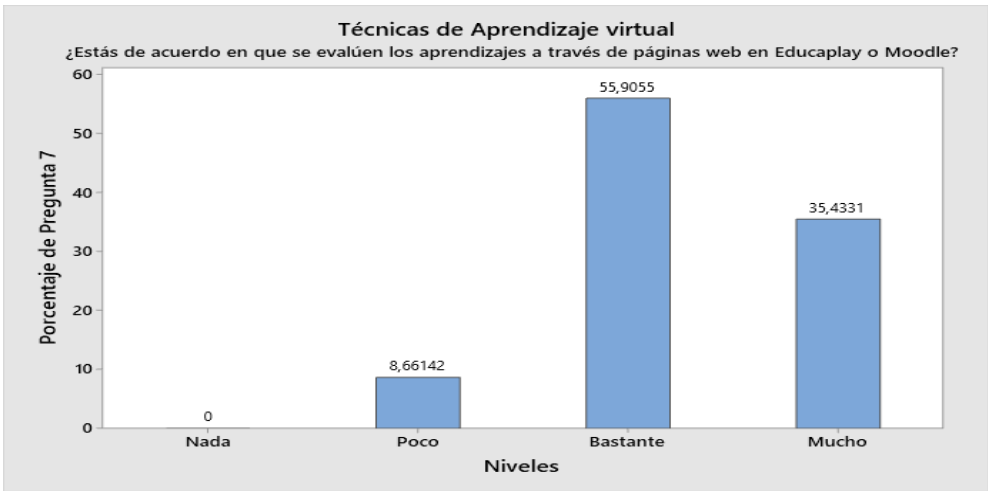

Fuente: Estudio de contexto 
En la gráfica de la pregunta 7 referente a: ¿Estás de acuerdo en que se evalúen los aprendizajes a través de páginas web en Educaplay o Moodle?, se observa claramente que el $0 \%$ de los estudiantes se encuentran en nada, de ahí el $8 \%$ de las personas están de acuerdo en la alternativa de poco, el 55\% opta por la opción de bastante y con el 35\% se tiene a la opción de mucho. Siendo así que, más del $50 \%$ de los estudiantes están de acuerdo en dicha pregunta, puesto a que dichas plataformas son útiles y prácticas para la modalidad virtual por la cual se está atravesando.

\section{Tabla 4}

Test de Dificultades Atencionales

\begin{tabular}{|c|c|c|c|c|c|c|c|c|}
\hline DESCRIPCION & \multicolumn{2}{|c|}{$\mathrm{NADA}=0 \%$} & \multicolumn{2}{|c|}{$\mathrm{POCO}=1 \%$} & \multicolumn{2}{|c|}{ BASTANTE $=2 \%$} & \multicolumn{2}{|c|}{$\mathrm{MUCHO}=3 \%$} \\
\hline $\begin{array}{l}\text { 1. Tienes excesiva } \\
\text { inquietud motora }\end{array}$ & 13 & $10 \%$ & 64 & $52 \%$ & 41 & $33 \%$ & 6 & $5 \%$ \\
\hline $\begin{array}{l}\text { 2. Tienes } \\
\text { dificultades de } \\
\text { aprendizaje escolar }\end{array}$ & 35 & $28 \%$ & 73 & $59 \%$ & 13 & $10 \%$ & 3 & $2 \%$ \\
\hline $\begin{array}{l}\text { 3.Molestas } \\
\text { frecuentemente a } \\
\text { otros estudiantes }\end{array}$ & 85 & $69 \%$ & 31 & $25 \%$ & 5 & $4 \%$ & 3 & $2 \%$ \\
\hline $\begin{array}{l}\text { 4. Te distraes } \\
\text { fácilmente, } \\
\text { muestras escasa } \\
\text { atención }\end{array}$ & 14 & $11 \%$ & 78 & $63 \%$ & 23 & $19 \%$ & 9 & $7 \%$ \\
\hline $\begin{array}{l}\text { 5.Exiges inmediata } \\
\text { satisfacción a tus } \\
\text { demandas }\end{array}$ & 11 & $9 \%$ & 64 & $52 \%$ & 42 & $34 \%$ & 7 & $6 \%$ \\
\hline $\begin{array}{l}\text { 6. Estás en las nubes, } \\
\text { ensimismado }\end{array}$ & 38 & $31 \%$ & 70 & $56 \%$ & 10 & $8 \%$ & 6 & $5 \%$ \\
\hline $\begin{array}{l}\text { 7. Dejas por terminar } \\
\text { las tareas que } \\
\text { empiezas }\end{array}$ & 33 & $27 \%$ & 49 & $40 \%$ & 29 & $23 \%$ & 13 & $10 \%$ \\
\hline $\begin{array}{l}\text { 8.Te mueves } \\
\text { constantemente, } \\
\text { intranquilo/a }\end{array}$ & 28 & $23 \%$ & 67 & $54 \%$ & 24 & $19 \%$ & 5 & $4 \%$ \\
\hline $\begin{array}{l}\text { 9. Eres impulsivo/a e } \\
\text { irritable }\end{array}$ & 43 & $35 \%$ & 52 & $42 \%$ & 22 & $18 \%$ & 7 & $6 \%$ \\
\hline $\begin{array}{l}\text { 10.Tus esfuerzos } \\
\text { se frustran } \\
\text { fácilmente, eres } \\
\text { inconstante }\end{array}$ & 29 & $23 \%$ & 72 & $58 \%$ & 18 & $15 \%$ & 5 & $4 \%$ \\
\hline $\begin{array}{c}\text { TOTAL AL } \\
100 \%\end{array}$ & & & & & & & & \\
\hline
\end{tabular}

Fuente: Encuesta virtual a estudiantes de la Universidad Técnica de Ambato 
Los estudiantes participantes en la investigación indicaron que tienen excesiva inquietud motora $10 \%$ nada, $52 \%$ poco, $33 \%$ bastante y $5 \%$ mucho. Tienen dificultades de aprendizaje escolar $28 \%$ nada, $59 \%$ poco, $10 \%$ bastante y $2 \%$ mucho. Molesta frecuentemente a otros estudiantes $69 \%$ nada, $25 \%$ poco, $4 \%$ bastante y $2 \%$ mucho. Los estudiantes se distraen fácilmente muestran escasa atención $11 \%$ nada, $63 \%$ poco, $19 \%$ bastante y $7 \%$ mucho. Exigen inmediata satisfacción a sus demandas $9 \%$ nada, 52 $\%$ poco, $34 \%$ bastante y $6 \%$ mucho. Los estudiantes indicaron que están en las nubes ensimismado $31 \%$ nada, $56 \%$ poco, $8 \%$ bastante y $5 \%$ mucho. Dejan por terminado las tareas que empiezan $27 \%$ nada, $40 \%$ poco, $23 \%$ bastante y $10 \%$ mucho. Se mueven constantemente, intranquilos $23 \%$ nada, $58 \%$ poco, $19 \%$ bastante y $4 \%$ mucho. Los estudiantes demostraron que sean impulsivos he irritables un $35 \%$ nada, $42 \%$ poco, $18 \%$ bastante y $6 \%$ mucho. Sus esfuerzos se frustran fácilmente y eres inconstante $23 \%$ nada, $58 \%$ poco, $15 \%$ bastante y $\%$ de mucho.

\section{Figura 3}

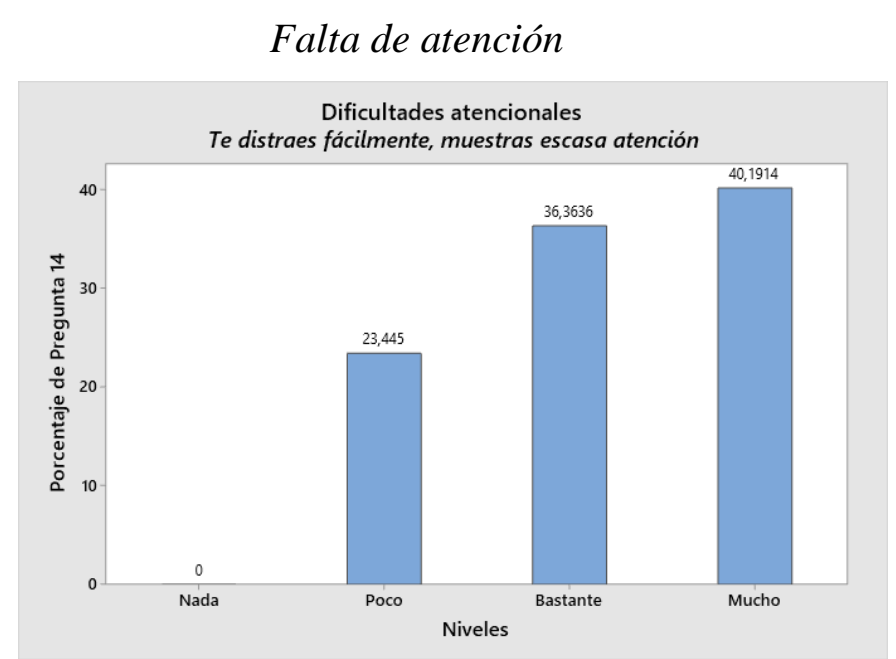

Fuente: Estudio de contexto

De acuerdo con la gráfica de la pregunta: Te distraes fácilmente, muestras escasa atención se muestra que el $0 \%$ de los estudiantes están en nada, de ahí el $23 \%$ poco se distrae con facilidad, seguidamente el $36 \%$ tienen mayor relevancia con la opción de bastante y el $40 \%$ concuerda en mucho, por los alumnos se distraen fácilmente, mostrando escasa atención durante las clases virtuales, lo que representa que poseen dificultad para concentrarse a lo que dice el maestro y por ende se ponen a realizar otras actividades extracurriculares. 


\section{Figura 4}

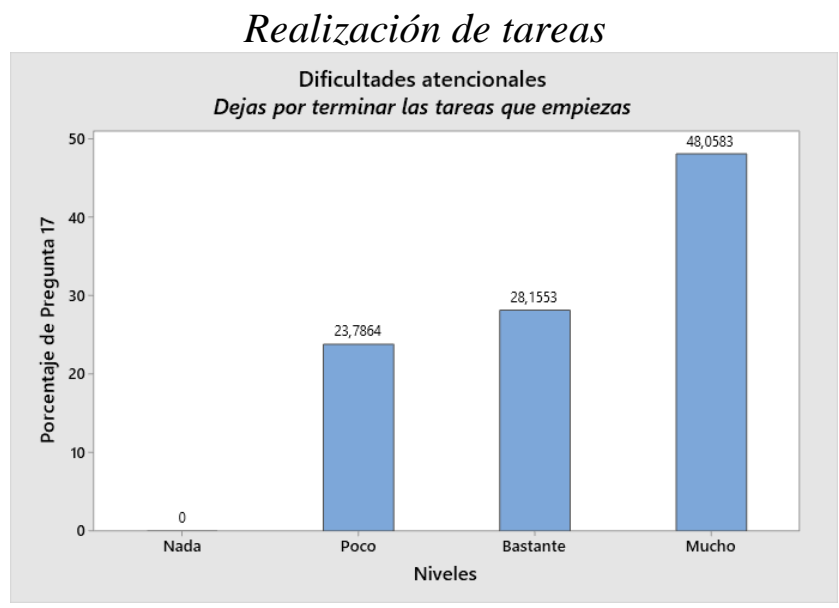

Fuente: Estudio de contexto

Con respecto a la gráfica de la pregunta: Dejas por terminar las tareas que empiezas, el $0 \%$ de los encuestados están en nada, el 23\% de los estudiantes se encuentran en la opción de poco, de ahí el $28 \%$ de los alumnos optaron por la alternativa de bastante y finalmente el $48 \%$ refleja que mucho de los estudiantes no acaban sus tareas que empiezan y no las culminan debido a diversos factores como pueden ser: falta de motivación, pierden con facilidad la atención o tienen muchos distractores.

\section{Figura 5}

\section{Gráfica de correlación entre las variables Técnicas de Aprendizaje virtual y Dificultades atencionales de Spearman}

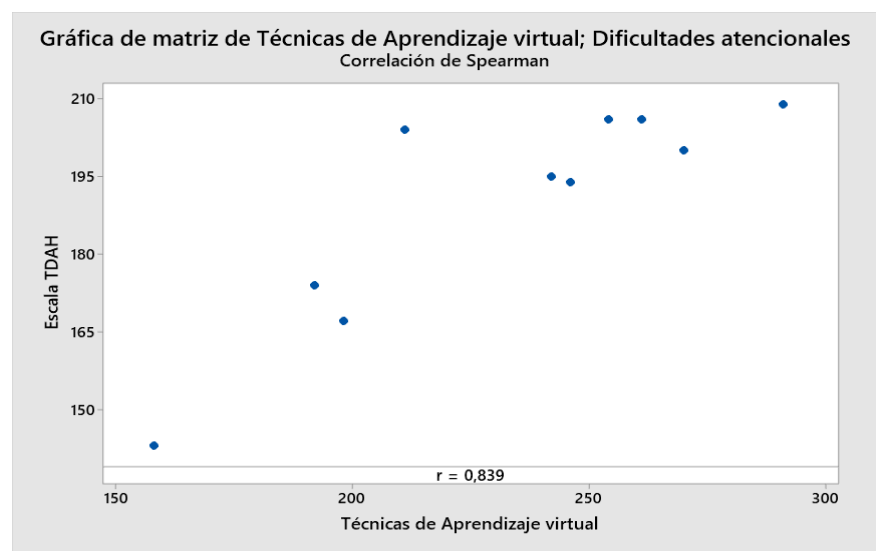

Fuente: Estudio de contexto

Se observa que la correlación de Spearman entre Técnicas de aprendizaje virtual y Dificultades atencionales es 0,839 lo que indica que existe una relación positiva entre las variables, lo que traduce en que si se emplean correctas actividades en clases por parte de los docentes y de igual manera si los estudiantes hacen uso de recursos tecnológicos que beneficien su estudio esto repercute en que disminuyan las dificultades atencionales en 
los alumnos.

\section{Conclusiones}

- Las técnicas de aprendizaje virtual influyen de manera significativa en los estudiantes con dificultades atencionales, puesto a que, si se emplean actividades que fomenten a la participación activa haciendo uso de las plataformas digitales y contenidos

- multimedia los cuales sean interactivos darán como resultado un mejor rendimiento académico en los educandos.

- Las dificultades atencionales se presentan por varias razones ya sean por factores externos o internos, dicho de otro modo, los distractores alrededor del espacio de trabajo del alumno y que las clases no cuenten con una metodología dinámica, son causantes de los estudiantes no enfoquen toda su atención lo que repercute en su proceso de adquirir conocimientos y destrezas para su formación.

- En definitiva, la implementación y aplicación de métodos adecuados por parte de los docentes durante las clases son necesarios para un correcto desempeño en los estudiantes, ya que estas permitirán la consolidación de información, además harán que el alumno se enfoque en lo que se está explicando y que sin duda se logre obtener resultados eficaces en el aprendizaje en línea.

\section{Referencias bibliográficas}

Abril Iza, M. (2020). El uso de la gamificación como estrategia didáctica en los niños con TDAH. http://repositorio.uti.edu.ec//handle/123456789/1522

$\begin{array}{lllll}\text { Aguilar Gordón, } & \text { F. } & \text { (octubre } & \text { de } & \text { 2020). }\end{array}$ https://scielo.conicyt.cl/pdf/estped/v46n3/0718-0705-estped-46-03-213.pdf

Asociación Americana de Psiquiatría. (2013). Gia de consulta de los Criterios Diagnósticos Del DSM-5. Arlington: American Psychiatric Publishing.

Cedeño Barreto, M. E., \& Rodríguez Gámez, M. (agosto de 2019). Trastorno por déficit de atención asociado o no a la hiperactividad y su impacto en el aprendizaje significativo. https://www.eumed.net/rev/caribe/2019/08/trastorno-deficitatencion.html

Chacón, O., Riaño, M., Bermudes, Quinteros Sangino, Hernández, M., \& Mendoza, M. (2018). ¿Es la obesidad un factor de riesgo para el trastorno de déficit de atención con hiperactividad (TDAH)? Revista Latinoamericana de Hipertensión, 1-12.

Cortes Bourne, C., \& Sánchez Salinas, S. (marzo de 2021). repositorio.ug.edu.ec. 
http://repositorio.ug.edu.ec/bitstream/redug/55655/1/TESIS\%20FINAL\%20CO RTE S\%20Y\%20S\%c3\%81NCHEZ\%20COMPLETO.pdf

Espinoza Freire, E. (2018). El tutor en los entornos virtuales de aprendizaje. Universidad y Sociedad. Universidad y Sociedad, 3. http://scielo.sld.cu/pdf/rus/v10n3/22183620-rus-10-03-201.pdf

Gavilanes, M., Yanza, W., Inca, A., Torres, G., \& Sánchez, R. (2019). Las Tics en los procesos de enseñanza y aprendizaje. Ciencia Digital, 8. https://cienciadigital.org/revistacienciadigital2/index.php/CienciaDigital/article/ vie $\mathrm{w} / 575 / 1382$

González, A., Ramos, A., \& Valeriana, P. (2020). Adolescentes TDAH: psicología y sexualidad. Universidad Católica de Colombia.

González, A., Pujol, F., Sánchez, Y., \& Sánchez, A. (2020). Manual de apoyo docente para la evaluación del aprendizaje mediante la utilización de medios de enseñanza. https://cienciadigital.org/revistacienciadigital2/index.php/exploradordigital/articl e/vi ew/1315/3225

Guerrero García, J., \& González Calleros, J. M. (marzo de 2021). Videojuegos en educación especial: niños con TDAH. Revista Digital de Asociación Interacción Persona-Ordenador, http://revista.aipo.es/index.php/INTERACCION/article/view/35/41

Jaime Torres, X. (2019). Páginas web educativas. Introducción, la Web en los ambientes educativos, educación y Web, ventajas y desventajas, diseño, tipos de información, herramientas para construir una página Web. 10.

Liesa, M., Cosculluela, C., \& Toledo, S. (2017). Habilidades sociales de niños con déficits atencionales y contextos escolares inclusivos. Revista Española de Orientación y Psicopedagogía, 113-121.

Martínez, D., \& Yévenes, S. (2020). Atención Durante la Pandemia COVID-19. International journal of odontostomatology, 1-14.

Martinhago, F., Lavagnino, N., Folguera, G., \& Caponi, S. (2019). Factores de riesgo y bases genéticas: el caso del trastorno por déficit de atención e hiperactividad. Salud Colectiva, 1-10.

Mattiooli, M. (2019). Los servicios de atención y educación de la primera infancia em américa latina. Análisis Comparativo de políticas de educación.

Navarrete, F., Sánchez, V., Altamirano, T., \& Cevallos, V. (2019). Utilización de aulas 
virtuales para el desarrollo de la inteligencia visual-espacial en estudiantes universitarios. Ciencia Digital, https://cienciadigital.org/revistacienciadigital2/index.php/CienciaDigital/article/ vie $\mathrm{w} / 785 / 1884$

Peña Estrada, C. (2020). Personas con discapacidad y aprendizaje virtual: Retos para las TIC en tiempos de Covid-19. Revista Tecnológica-Educativa, 5.

Quila, R., Luna, P., Suarez, D., \& Pérez, M. (2020). Increíbles estrategias de aprendizaje creativo para formar estudiantes con responsabilidad social en el siglo XXI. Ciencia

Digital,

5. https://cienciadigital.org/revistacienciadigital2/index.php/ConcienciaDigital/arti cle/ view/1358/3348

Ramírez, A., Yaneth, O., \& Milena, A. (2020). Dificultades atencionales y habilidades académicas en estudiantes.

Restrepo Echavarría, R. (agosto de 2018). Aprendizaje activo para el aula: Una síntesis de fundamentos y técnicas. https://unae.edu.ec/wpcontent/uploads/2019/11/cuaderno- 2.pdf

Suarez, S., Ruiz-Ariza, A., López-Serrano, S., \& Martínez López, E. (2018). Descansos activos para mejorar la atención en clase: intervenciones educativas. Profesorado, Revista de Currículum y Formación del Profesorado, 287-304.

Valero Cedeño, N. (2020). Retos de la educación virtual en el proceso enseñanza aprendizaje durante la pandemia de Covid-19. Dominio de las Ciencias, 7-9.

Valverde, G., Almeida, E., Mayko, S., \& Jiménez, M. (2020). Educación emocional y su incidencia en el aprendizaje de Ciencias Naturales en niños con TDAH. Horizontes Revista de Investigación en Ciencias de la Educación.

\section{Ciencia Ligital}


El artículo que se publica es de exclusiva responsabilidad de los autores y no necesariamente reflejan el pensamiento de la Revista Conciencia Digital.

\section{Ciencia \\ LDigital}

El artículo queda en propiedad de la revista y, por tanto, su publicación parcial y/o total en otro medio tiene que ser autorizado por el director de la Revista Conciencia Digital.

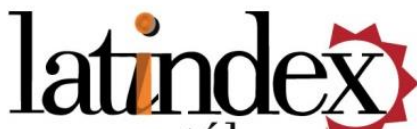

catálogo

ठ

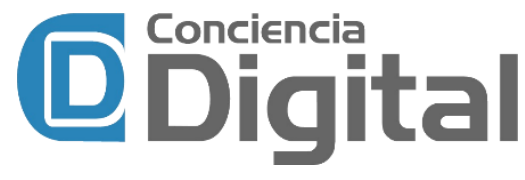

Indexaciones doi crossref latindex̃

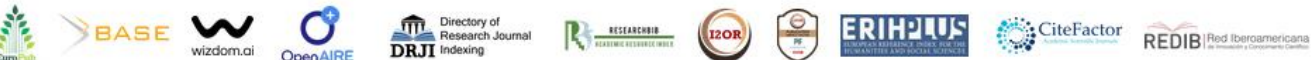

